\title{
Ventajas del reforzamiento de muros de bloques de tierra compactados (BTC), como opción para el rescate de viviendas rurales
}

\author{
Advantages of reinforcing compressed soil block (BTC) walls \\ as a form to preserve the rural housing
}

L. A. Juárez $z^{(*)}$, T. Caballero ${ }^{(*)}$, V. Morales ${ }^{(*)}$

\section{RESUMEN}

Se realizó una investigación experimental en pilas de bloques de tierra compactados (BTC) reforzadas con malla hexagonal y malla electrosoldada para determinar la influencia del refuerzo, mediante ensayes de compresión axial, compresión diagonal y flexión lateral, según lo especifican las Normas Técnicas Complementarias para Mampostería (NTCM ${ }^{(1)}$ y UNAM $^{(2)}$ ) del DF, en México. En el ensayo a compresión axial, se determinó que la carga máxima admisible se incrementó significativamente en el grupo reforzado con malla electrosoldada (39\%), no así la resistencia $(4 \%)$. En el ensaye a compresión diagonal, el incremento en la carga máxima admisible y resistencia fue significativo para los dos grupos reforzados, de 90 a $125 \%$ y de 60 a $83 \%$ respectivamente. A flexión lateral los incrementos fueron altamente significativos para el grupo reforzado con malla electrosoldada. Aunque se determinaron los parámetros de diseño a compresión, cortante y flexión del BTC, es importante realizar mas ensayes para corroborar estos resultados.

\section{$471-5$}

Palabras Clave: bloque de tierra compactado, albañilería, reforzamiento, resistencia a compresión, resistencia de la mampostería.

\section{SUMMARY}

Experimental research was made in compressed soil block (BTC) piles. These specimens were reinforced with hexagonal mesh and welded wire mesh to determinate its mechanics characteristics by means of axial and diagonal compression, and flexion tests. We made experimental assays in accord with Mexican Code specifications of $\mathrm{NTCM}^{(1)}$ and $\mathrm{UNAM}^{(2)}$ Engineering Institute researchers. In axial compression test, the piles reinforced with welded wire mesh had a significant increase in maxim admissible load (39\%) but had not in masonry strength (4\%). In diagonal compression test, the reinforced groups had significant increases in both, load capacity (90-125\%) and masonry strength (60-83\%). The strength and deformation capacity were higher in specimens reinforced with welded wire mesh. In the flexion test had similar results. Although the compression, shear and flexion design parameters was determinate, it's important and necessary to make more tests to corroborate these results.

Keywords: compressed soil block, masonry, reinforces, compression strength, masonry strength.
(1) Normas Técnicas Complementarias para Diseño y Construcción de Mampostería.

(2) Universidad Nacional Autónoma de México. 
Notaciones:

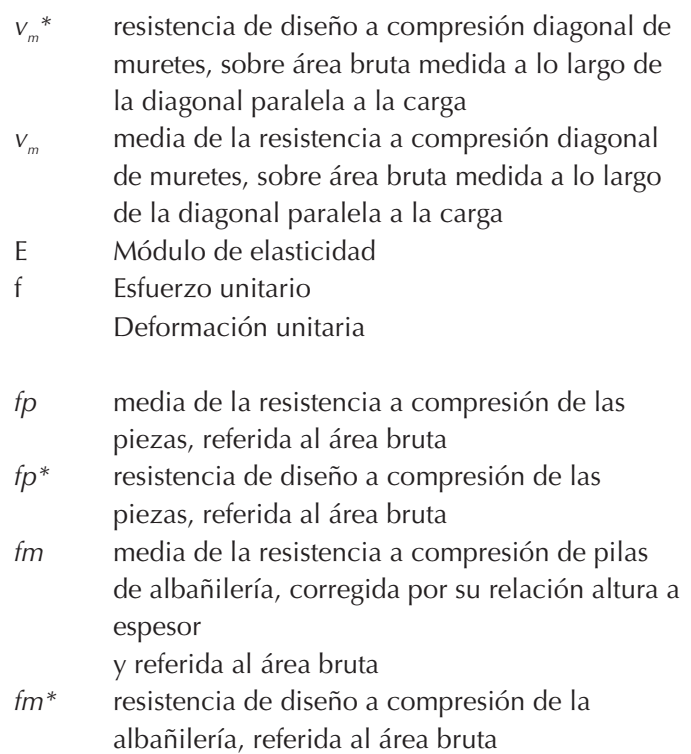

$P$

carga axial total que obra sobre el muro

G Módulo cortante de elasticidad

$v \quad$ Esfuerzo cortante unitario

$\gamma \quad$ Deformación cortante unitaria

ff Media de la resistencia a flexión de los muretes.

ff* Resistencia de diseño a flexión de la albañilería, referida al área bruta

Gm módulo de cortante de la albañilería

Em módulo de elasticidad de la albañilería para esfuerzos de compresión normales a las juntas

CV Coeficiente de variación

\section{INTRODUCCIÓN}

La vivienda es una necesidad básica de los seres humanos y es un indicador de las condiciones de vida de la población. Los materiales utilizados en su construcción son variados. En el estado de Oaxaca (México), la mayor parte de la población se localiza en comunidades menores a 2.500 habitantes y de acuerdo a los datos estadísticos del más reciente Censo de Población y Vivienda, el $24 \%$ de las viviendas existentes en el Estado tienen muros a base de adobe (1).

La vivienda rural generalmente es de adobe, material elaborado en forma tradicional, lo cual lo hace vulnerable ante eventos naturales como son la lluvia y los sismos. Sin embargo, por las características culturales, sociales y económicas del medio rural en Oaxaca (México) sigue constituyendo un material de uso amplio en la construcción de viviendas. Los bloques de tierra compactados (BTC) constituyen una alternativa económica y segura para la construcción de viviendas, es un material de construcción que presenta ventajas sobre el adobe tradicional, conservando sus características de confort e incrementando su resistencia mecánica (2). Sin embargo, por su comportamiento ante eventos sísmicos (3) se consideró conveniente aplicar un reforzamiento a la albañilería de adobe para mejorar su comportamiento ante la acción de las fuerzas dinámicas que se generan durante un sismo y darle una mayor vida útil mediante la aplicación de un recubrimiento, tomando en consideración los costos y tipos de materiales disponibles. El reforzamiento de muros de adobe y BTC en viviendas, es un tema de importancia, por el número considerable de viviendas que se construyen mediante este sistema (4); sin embargo, particularmente sobre el BTC existen muy pocos estudios relacionados con su comportamiento mecánico y sobre su reforzamiento para incrementar su resistencia ante diversos agentes externos. En el estado de Oaxaca, México se tiene un alto grado de sismicidad por lo que el factor seguridad es un elemento muy importante a considerar en el uso de nuevos materiales, como el BTC.

La baja resistencia a la tensión de la albañilería limita fuertemente la capacidad de los muros para resistir cargas laterales. Una de las técnicas de rehabilitación aplicable a muros de mampostería es el encamisado de muros, que consiste en adosar mallas metálicas al mismo, adecuadamente ancladas, recubriéndolas con mortero de cemento. En el estudio de muros de BTC existe gran número de variables que es necesario tomar en cuenta y algunas de ellas, como las propiedades de los materiales, la calidad de la mano de obra, las condiciones de contacto entre el muro y el marco o la adherencia entre mortero y piezas, son muy difíciles de controlar y/o cuantificar (5).

En el presente trabajo se determinó cuantitativamente la variación en la resistencia de los muros de BTC sujetos a cargas de compresión directa, compresión diagonal y flexión, reforzados con mallas de acero y recubiertos con mortero cemento-arena, empleando materiales locales. Las mallas utilizadas y el mortero se seleccionaron con base en la experiencia del grupo de investigación que ha desarrollado otras edificaciones con materiales similares. En las consideraciones de 
diseño del experimento se tomó en cuenta la facilidad, rapidez, costo y seguridad para colocar las mallas, así como la disponibilidad en el mercado.

Los ensayos se realizaron de acuerdo a lo especificado en las Normas Técnicas Complementarias para el Diseño y Construcción de Mampostería (6) del DF y por investigadores del Instituto de Ingeniería de la Universidad Nacional Autónoma de México (UNAM).

\section{PROCEDIMIENTO EXPERIMENTAL}

La metodología de este estudio experimental fue el siguiente:

- Elaboración de las piezas de BTC

- Determinación de sus características

- Selección del tipo de refuerzo

- Construcción de probetas:

- Pilas para ensayo a compresión axial (ver Figura 1)

- Muretes para ensayo a compresión diagonal $(\mathrm{L} 1 / \mathrm{L} 2=1)$

- Muretes, para ensayo a flexión

- Realización de ensayos en probetas reforzadas y en grupo control:

-Compresión axial

-Compresión diagonal

-Flexión

- Análisis estadístico de resultados

- Contrastación de resultados

Las piezas empleadas se caracterizaron mediante el ensayo de 90 piezas provenientes del muestreo de dos lotes de producción de BTC elaborados con tierra cruda estabilizada con cemento $(8 \%)$ y compactados con una prensa mecánica, similar a la máquina CINVA RAM.

Se determinaron sus características principales como son: dimensiones, peso volumétrico, absorción y resistencia a la compresión, de acuerdo con los ensayos especificados en la normas NMX-C-036-ONNCCE-2004 (7), NMX-C-037-ONNCCE-2005 (8), NMXC-038-ONNCCE-2004 (9).

Posteriormente se construyeron 81 pilas y muretes para determinar su resistencia a la compresión en pila, compresión diagonal y flexión lateral. En los apartados 2.8.1 y 2.8.2. de las NTCM (6) se especifica cómo determinar la resistencia a compresión axial y diagonal del muro mediante ensayos de pilas, las cuales se deberán realizar en un mínimo de nueve pilas. Los modelos para compresión axial fueron construidos en forma continua con una altura de seis piezas, con una relación altura-espesor promedio de cuatro y, para compresión diagonal, la base estaba formada por una y media piezas y la altura por cinco piezas (relación L1=L2), sujetas con mortero cemento-cal-arena en proporción 1:0.25:2.5, elaborado mecánicamente, bajo diseño previo. En la Figura 1 se muestra la forma y aplicación de carga en las pilas de ensayo a compresión axial (a) y diagonal (b). A uno de los grupos se les colocó el refuerzo con malla hexagonal de 1" de abertura y alambre calibre 22 sujeta con clavos de $21 \frac{1}{2}$ " distribuidos uniformemente; al otro se les colocó el refuerzo de malla electrosoldada 6 × 6 - 10/10 sujeta con cinco anclas de alambrón de 1/4" colocadas a la altura de las juntas y de acuerdo a lo especificado en el inciso 3.3.6.5. de las NTCM (6); se cubrió con mortero cemento-arena en proporción volumétrica 1:2.5 elaborado mecánicamente, bajo diseño previo, con una resistencia promedio de $37,3 \mathrm{MPa}\left(373 \mathrm{~kg} \mathrm{\textrm {cm } ^ { - 2 }}\right)$. Se construyó un tercer grupo sin refuerzo como control.

En el apartado 3.1.7 de las NTCM (6) se hace referencia sobre la resistencia de la mampostería sujeta a cargas laterales, y se establece que la fuerza cortante que toma la mampostería se basa en el esfuerzo cortante resistente de diseño que en esta Norma es igual a la resistencia a compresión diagonal $\left(\mathrm{vm}^{*}\right)$. En este estudio se consideró conveniente realizar un experimento específico para determinar la resistencia de los muretes de BTC a flexión lateral, implementando un dispositivo mecánico que permitiera la aplicación uniforme de carga lateral al murete, sin ocasionar fallas locales (Figura 2). El ensayo a flexión lateral se basó en la teoría des-

Pilas para ensayos a compresión.

Dispositivo y colocación de murete para el ensayo a flexión lateral.
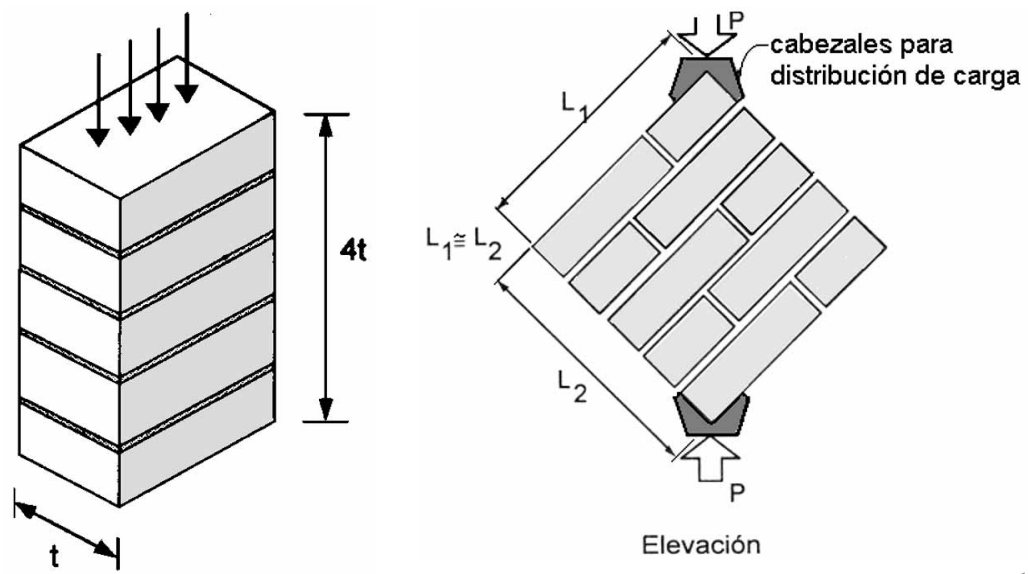

Elevación
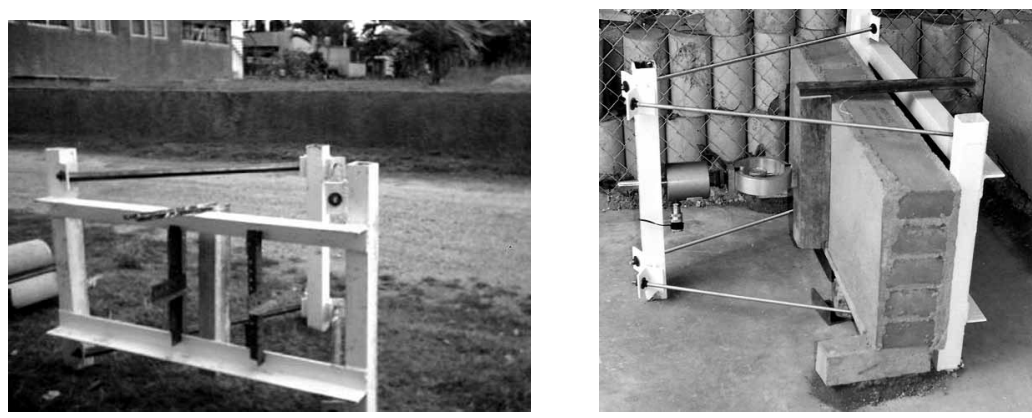

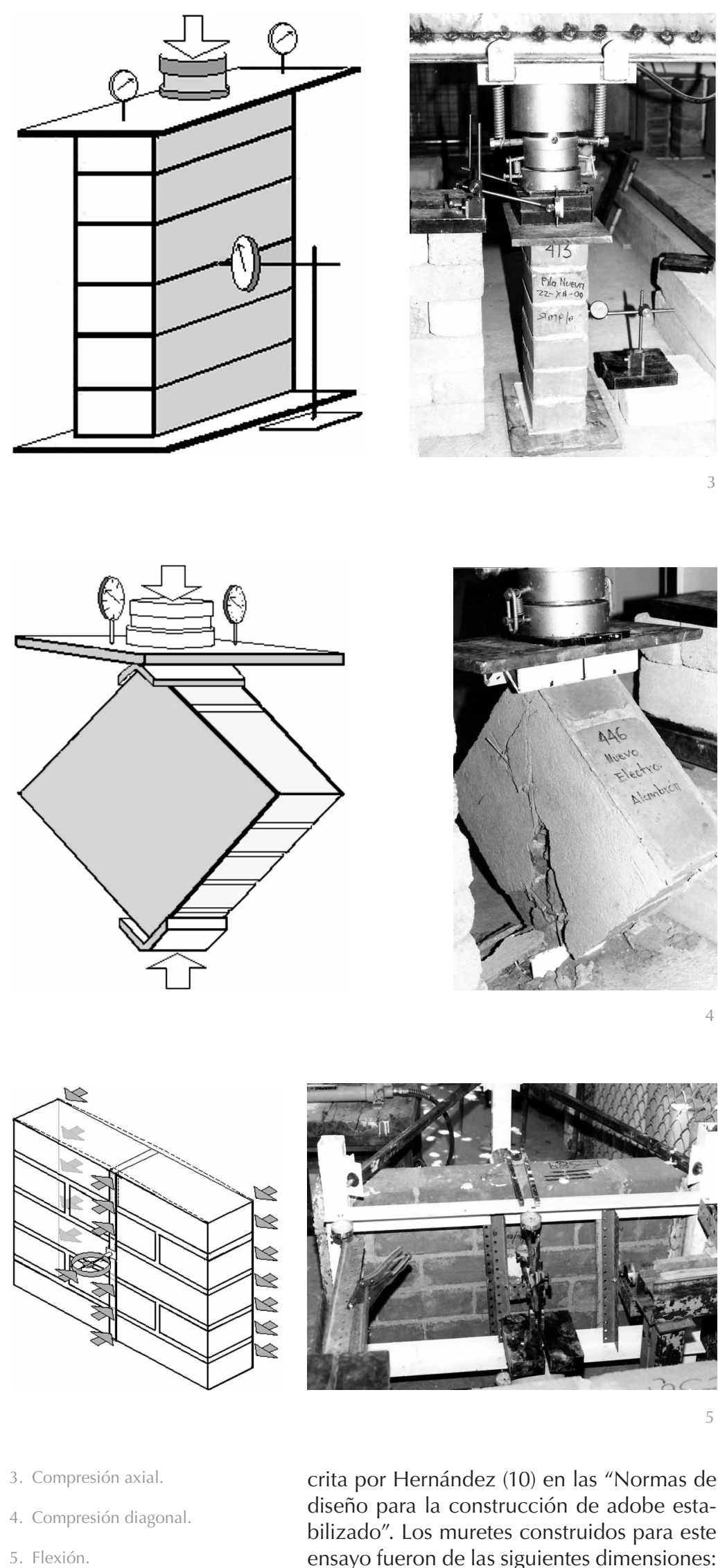

crita por Hernández (10) en las "Normas de diseño para la construcción de adobe estabilizado". Los muretes construidos para este ensayo fueron de las siguientes dimensiones: $1,20 \mathrm{~m}$. de longitud y cinco hiladas de altura; se utilizó mortero cemento-cal-arena elaborado mecánicamente, en proporción 1:0.25:2.5 para el junteo, reforzados en forma similar a los ensayos de compresión.
Cuando un material está sometido a fuerzas externas, desarrollará uno o más de los siguientes tipos de deformación: elástica lineal, elástica no lineal, visco elástica, plástica inelástica. Muchos materiales estructurales tienen deformaciones lineales elásticas bajo cargas de diseño; para estos materiales la deformación unitaria $\varepsilon$, es proporcional al esfuerzo unitario $f$, hasta que se excede cierto esfuerzo, o sea, el límite de proporcionalidad, esta relación [1] se conoce como Ley de Hooke (11). Para las cargas axiales de tensión o compresión, la relación se puede escribir:

$f=E \varepsilon$

donde:

E es el módulo de elasticidad del material.

En el ensayo a compresión axial las deformaciones de las pilas se midieron a través de tres micrómetros de 0,001 mm de aproximación, colocando dos de ellos en la parte superior de la pila y el tercero en la parte lateral de la misma, según se muestra en la Figura 3, en el ensayo a compresión diagonal las deformaciones se midieron mediante la colocación de dos micrómetros de 0,001mm de aproximación ubicados sobre una placa de acero colocada en la parte superior de la pila, según se muestra en la Figura 4 y en la Figura 5 se muestra la colocación de los micrómetros en la prueba de flexión.

\section{RESULTADOS}

En la Tabla 1 se resumen los resultados de la caracterización de piezas de BTC provenientes de dos lotes de producción. Como se encontró variabilidad en los resultados, se analizaron estadísticamente aplicando tstudent, determinando que los datos de resistencia a compresión para los dos lotes de producción pueden considerarse como una misma población, no siendo así en el peso volumétrico y la resistencia a flexión. El valor promedio de resistencia a la compresión fue de 9,12 $\mathrm{MPa}\left(91,19 \mathrm{~kg} \mathrm{~cm}^{-2}\right)$ y resistencia a la flexión de 0,59 MPa $\left(5,95 \mathrm{~kg} \mathrm{~cm}^{-2}\right)$.

Los resultados del ensayo a compresión axial se presentan en la Tabla 2, donde se aprecia un incremento mayor en carga máxima admisible (39\%) y esfuerzo (4\%) en el grupo reforzado con malla electrosoldada.

Tomando como referencia la resistencia promedio de las piezas, $\mathrm{fp}=9.12 \mathrm{MPa}$ $\left(91,19 \mathrm{~kg} \mathrm{~cm}^{-2}\right)$ se determinó la relación con la resistencia promedio de las pilas: $\mathrm{fm}($ sin refuerzo $)=0,323 \mathrm{fp}$. Los resultados del ensayo a compresión diagonal se presentan en la Tabla 3, donde se aprecia un incremento notable tanto en carga máxima 
Tabla 1

Características de los (BTC) bloques de tierra compactados (12)

\begin{tabular}{|c|c|c|c|c|c|c|}
\hline \multirow{2}{*}{ 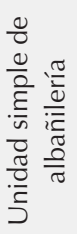 } & \multirow{2}{*}{ 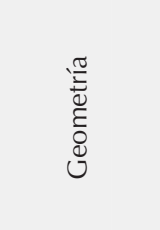 } & \multirow{2}{*}{$\begin{array}{ll}0 & \frac{0}{0} \\
0 & \frac{0}{0} \\
0 & \frac{0}{0} \\
0 & 0 \\
0 & 0 \\
& 0\end{array}$} & \multirow{2}{*}{$\begin{array}{l}0^{\circ} \\
\frac{0}{0} \\
\frac{0}{0} \\
0 \\
0 \\
\frac{0}{<}\end{array}$} & \multirow{2}{*}{ 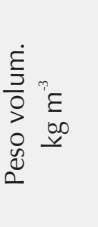 } & \multicolumn{2}{|c|}{$\begin{array}{c}\text { Resistencia a compresión } \\
\qquad \mathrm{MPa}\end{array}$} \\
\hline & & & & & Media & $\mathrm{CV}$ \\
\hline & & 1 & ND & 1767.1 & 10.18 & 44.76 \\
\hline BTC & $9 \times 14 \times 28 \mathrm{~cm}$ & 2 & 16.45 & 1835.9 & 8.29 & 30.41 \\
\hline & & 1,2 & & 1805.3 & 9.12 & 40.13 \\
\hline
\end{tabular}

Tabla 2

Resultados del ensayo a compresión axial (12)

\begin{tabular}{|l|ccccc|cccc|}
\hline $\begin{array}{c}\text { Ensayo: } \\
\begin{array}{c}\text { Resistencia a } \\
\text { compresión axial }\end{array}\end{array}$ & $\begin{array}{c}\text { Carga } \\
\text { Prom } \\
(\mathrm{T})\end{array}$ & $\begin{array}{c}\text { Desviación } \\
\text { estándar }\end{array}$ & $\mathrm{CV}$ & $\begin{array}{c}\text { Incremento } \\
\%\end{array}$ & $\begin{array}{c}\text { Esfuerzo } \\
\text { Prom } \\
\text { (Mpa) }\end{array}$ & $\begin{array}{c}\text { Desviación } \\
\text { estándar }\end{array}$ & $\begin{array}{c}\text { CV } \\
\text { Incremento } \\
\%\end{array}$ \\
\hline M. HEXAGONAL & 14.41 & 2.32 & 16.1 & 8 & 2.62 & 0.48 & 0.18 & -11 \\
\hline M. ELECTROSOLDADA & 18.5 & 4.81 & 25.9 & 39 & 3.06 & 0.73 & 0.24 & 4 \\
\hline SIN REFUERZO & 13.32 & 2.47 & 18.6 & 0 & 2.94 & 0.53 & 0.18 \\
\hline
\end{tabular}

Tabla 3

Resultados del ensayo a compresión diagonal (12)

\begin{tabular}{|c|c|c|c|c|c|c|c|c|c|c|}
\hline $\begin{array}{l}\text { Ensayo: } \\
\text { Resistencia a cortante }\end{array}$ & $\begin{array}{c}\text { Carga } \\
\text { Prom } \\
(\mathrm{T})\end{array}$ & $\begin{array}{c}\text { Desviación } \\
\text { estándar }\end{array}$ & C & & $\begin{array}{c}\text { Incremento } \\
\%\end{array}$ & $\begin{array}{c}\text { Esfuerzo } \\
\text { Prom } \\
\text { (Мра) }\end{array}$ & $\begin{array}{l}\text { Desviación } \\
\text { estándar }\end{array}$ & \multicolumn{2}{|c|}{ CV } & $\begin{array}{c}\text { Incremento } \\
\%\end{array}$ \\
\hline M. HEXAGONAL & 6.74 & 0.90 & 13.41 & 0.20 & 91 & 0.57 & 0.08 & 13.43 & 0.20 & 59 \\
\hline M. ELECTROSOLDADA & 7.93 & 1.02 & 12.91 & 0.20 & 125 & 0.66 & 0.09 & 13.35 & 0.20 & 83 \\
\hline SIN REFUERZO & 3.52 & 1.56 & 44.25 & 0.44 & 0 & 0.36 & 0.16 & 44.91 & 0.45 & 0 \\
\hline
\end{tabular}

admisible como en esfuerzo para los dos grupos reforzados.

Tomando como referencia la resistencia promedio de pilas a compresión axial, $\mathrm{fm}=2,94$ MPa (29,45 $\left.\mathrm{kg} \mathrm{cm}^{-2}\right)$, se determinó la relación con la resistencia a cortante vm (12).

$\mathrm{vm}$ (muros sin refuerzo) $\sim 0,125 \mathrm{fm}$ $\mathrm{vm}$ (muros reforzados) $\sim 0,20-0,22 \mathrm{fm}$.

En el ensayo a compresión axial, el grupo con malla electrosoldada presentó un comportamiento lineal, sin llegar al colapso total de la probeta, alcanzando una resistencia máxima de $4.0 \mathrm{MPa}\left(40 \mathrm{~kg} \mathrm{~cm}^{-2}\right)$ y una deformación unitaria de 0.0021 (Figura 6, pág. siguiente).
El módulo de elasticidad "E" de un material está definido por la pendiente de la recta en la cual la deformación unitaria es proporcional al esfuerzo, esto es, la curva esfuerzo-deformación unitaria presenta un comportamiento lineal, definido por limites de proporcionalidad. El módulo de elasticidad para el BTC determinado a partir de las graficas esfuerzo-deformación unitaria, cuyos límites de proporcionalidad se definieron entre el 15 y $50 \%$ de la resistencia, fue de 793,4 $\mathrm{MPa}$ $\left(7.934 \mathrm{~kg} \mathrm{~cm}^{-2}\right.$ ).

El módulo de rigidez G o módulo cortante de elasticidad, ec. [2], se define por la relación entre el esfuerzo cortante unitario $v$ y la deformación cortante unitaria $n$, 

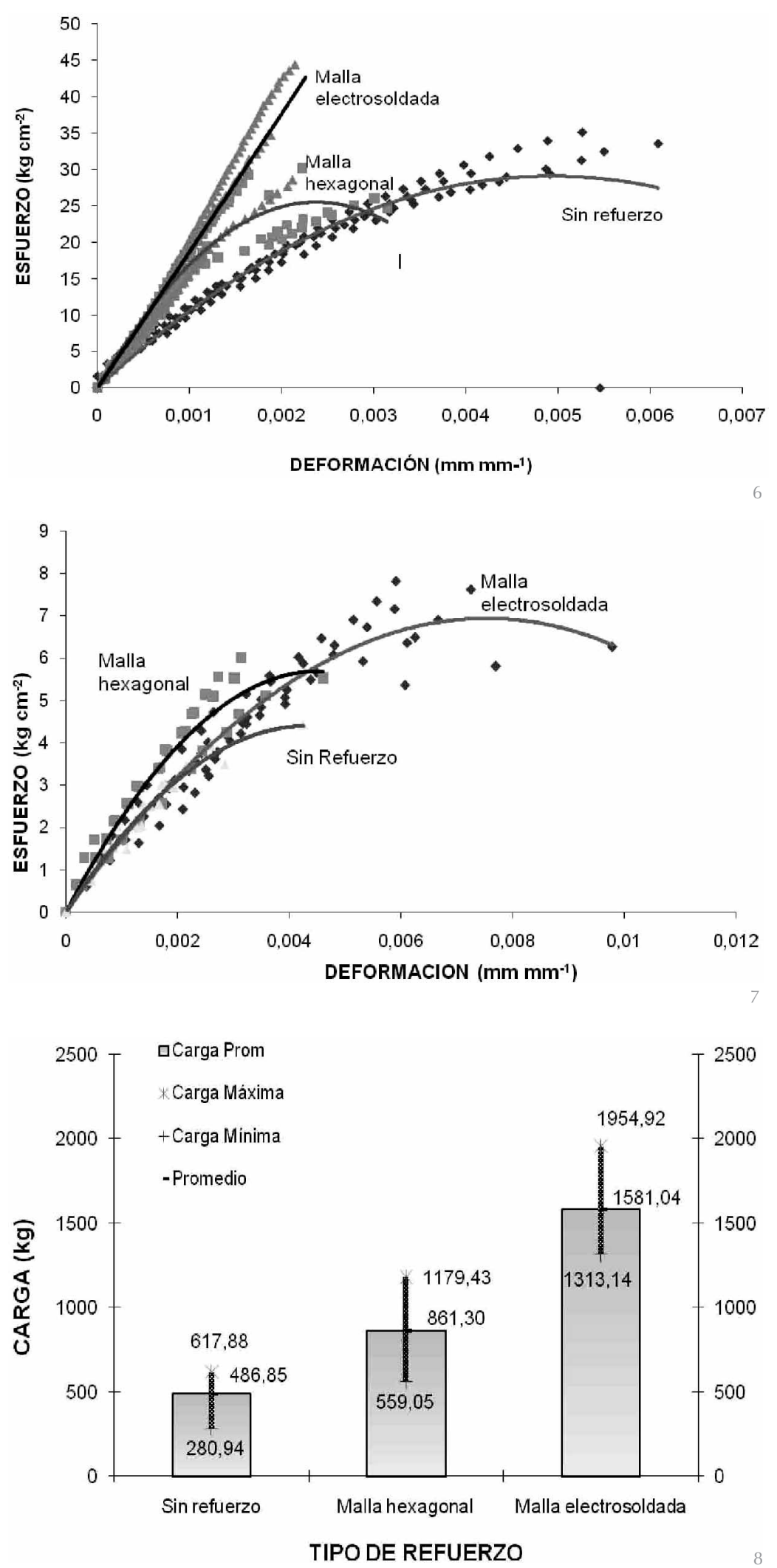

6. Relación esfuerzo-deformación ensayo a compresión axial.

7. Relación esfuerzo-deformación ensayo a compresión diagonal.

8. Carga máxima admisible, ensayo a flexión. con un valor promedio de 187.1 MPa (1.871 $\mathrm{kg} \mathrm{cm}^{-2}$ ). En la Figura 7 se observa el incremento en la capacidad de deformación de las pilas y en la resistencia, para los tres grupos (12).

En el ensayo a la flexión lateral tanto la capacidad de carga como la resistencia de los muretes reforzados presentaron incrementos significativos con respecto al grupo control, principalmente con la malla electrosoldada. En la Figura 8 se presenta la gráfica con la carga máxima soportada por los muretes en las tres series de ensayos y en la Tabla 4 se presentan los resultados de carga máxima admisible y resistencia de los muretes, calculada a partir de la ecuación [3] dada por Hernández (10):

$f_{f}=\frac{3 P l}{2 h t^{2}}$

donde:

$\mathrm{P}=$ carga que produjo la falla

$\mathrm{I}=$ longitud entre apoyos

$\mathrm{h}=$ altura del murete

$\mathrm{t}=$ espesor

En forma resumida tenemos en la Tabla 5 los resultados de los tres ensayos realizados, cuyas correlaciones muestran el grado de significancia del reforzamiento de pilas y muretes, con relación al grupo control (12). Se considera que los resultados de este trabajo contribuyen al conocimiento del comportamiento de la albañilería de BTC bajo diversas acciones de carga, incluido el comportamiento ante sismos, aunque se determinaron resistencias de diseño, es necesario realizar más ensayos para corroborar los resultados.

\section{DISCUSIÓN}

Los resultados en los dos lotes de BTC muestran una variabilidad elevada, pero dentro del rango de aceptabilidad, similar a la encontrada por Meli y Hernández (13) en piezas de tabiques y bloques, quienes analizaron muros construidos con diferentes tipos de piezas: tabique rojo recocido, tabicón, tabique extruido, y bloque de concreto hueco y macizo. Los resultados en tabique rojo recocido presentan coeficientes de variación que van del 20 al $36 \%$, una relación $\mathrm{fm} / \mathrm{fp}$ $=0,28-0,62$, y $\mathrm{E} / \mathrm{fm}=200-360$, con resistencias $\mathrm{fm}=1,2-5,6 \mathrm{MPa}\left(12-56 \mathrm{~kg} \mathrm{~cm}^{-2}\right)$ y $\mathrm{fp}=4.3-11.2 \mathrm{MPa}\left(43-112 \mathrm{~kg} \mathrm{~cm}^{-2}\right)$; mientras que en el BTC los coeficientes de variación fueron de 30 y $45 \%$, valores aceptables ya que en las Normas técnicas complementarias se menciona que el coeficiente de variación para el cálculo de la resistencia de diseño no será menor que 35\% para piezas de producción artesanal. En cuanto a las relaciones existentes entre los valores de diseño, 
Tabla 4

Carga máxima admisible y resistencia de muretes en ensayo a flexión

\begin{tabular}{|l|lccc|cccc|}
\hline $\begin{array}{l}\text { Ensayo: } \\
\text { Resistencia a flexión } \\
\text { lateral }\end{array}$ & $\begin{array}{l}\text { Carga } \\
\text { prom. } \\
(\mathrm{kg})\end{array}$ & $\begin{array}{c}\text { Desviación } \\
\text { estándar }\end{array}$ & $\mathrm{CV}$ & $\begin{array}{c}\text { Incremento } \\
\%\end{array}$ & $\begin{array}{c}\text { Resistencia } \\
\text { prom. } \\
(\mathrm{MPa})\end{array}$ & $\begin{array}{c}\text { Desviación } \\
\text { estándar }\end{array}$ & $\begin{array}{c}\text { Incremento } \\
\%\end{array}$ \\
\hline M. HEXAGONAL & 794.88 & 202.69 & 0.26 & 85 & 0.80 & 0.20 & 0.25 & 19 \\
\hline M. ELECTROSOLDADA & 1669.09 & 174.47 & 0.10 & 288 & 1.63 & 0.19 & 0.12 & 136 \\
\hline SIN REFUERZO & 430.69 & 111.40 & 0.26 & 0 & 0.68 & 0.17 & 0.25 & 0 \\
\hline
\end{tabular}

Tabla 5

Resultados de ensayos a pilas y muretes de BTC

\begin{tabular}{|c|c|c|c|c|c|c|c|c|c|}
\hline \multirow{2}{*}{$\begin{array}{c}\mathrm{f}_{\mathrm{p}}^{*}=4.5 \mathrm{MPa} \\
\left(45 \mathrm{~kg} \mathrm{~cm}^{-2}\right)\end{array}$} & \multicolumn{3}{|c|}{$\begin{array}{c}\text { Carga axial total, } \mathrm{P} \\
\text { (T) }\end{array}$} & \multicolumn{3}{|c|}{$\begin{array}{l}\text { Esfuerzos } \\
\text { (MPa) }\end{array}$} & \multicolumn{3}{|c|}{ Resistencia de diseño (MPa) } \\
\hline & Compresión & Cortante & Flexión & $f_{m}$ & $\mathrm{v}_{\mathrm{m}}$ & $f_{f}$ & $f^{*}{ }_{m}$ & $\mathrm{~V}_{\mathrm{m}}^{*}$ & $f_{f}^{*}$ \\
\hline SIN REFUERZO & 13.318 & 3.52 & 0.431 & 2.94 & 0.36 & 0.68 & 2.05 & 0.17 & 0.42 \\
\hline M. HEXAGONAL & 14.40 & 6.74 & 0.795 & 2.62 & 0.57 & 0.80 & 1.65 & 0.38 & 0.49 \\
\hline M. ELECTROSOLDADA & 18.498 & 7.93 & 1.669 & 3.06 & 0.66 & 1.60 & 1.98 & 0.44 & 1.24 \\
\hline
\end{tabular}

también se comprobó que la resistencia de las pilas esta en función de la resistencia de las piezas; el lote 1 presenta mayor resistencia que el lote 2 , la relación de fm / fp $=0,32$ para los dos lotes de producción y el módulo de elasticidad Em = 240 fm (lote 1). Alcocer (14) menciona que en ensayos recientes se ha demostrado que vm es directamente proporcional a la resistencia a compresión y a la retentividad del mortero.

En los ensayos realizados al BTC se corroboró esta afirmación, pues se encontró una relación directamente proporcional de $\mathrm{vm}=$ $0,3 \mathrm{fm}$ para las pilas de BTC, y en los muretes reforzados se encontró $\mathrm{vm}=0,15 \mathrm{fm}$ (malla hexagonal) y $\mathrm{vm}=0,11 \mathrm{fm}$ (malla electrosoldada). En cuanto a la rigidez, se ha comparado el módulo de rigidez al corte Gm medido en ensayos de compresión diagonal con el módulo de elasticidad Em obtenido en pilas.

Los resultados indican que el cociente $\mathrm{Gm} /$ Em varía entre 0,1 para piezas de alta resistencia y 0,3 para piezas más débiles. Valor adoptado generalmente en reglamentos de diseño (12). El valor promedio de Em encontrado para muretes de BTC fue de 7934 y el de Gm de 1871 lo cual da una relación G =
0,236 E. En el caso de muretes reforzados la relación es $\mathrm{G}=0,12 \mathrm{E}$ usando malla hexagonal, y $\mathrm{G}=0,08 \mathrm{E}$ usando malla electrosoldada. Estos valores se encuentran dentro de los valores mencionados por Alcocer (14), tanto para piezas de alta resistencia, como débiles, si tomamos en consideración que las probetas reforzadas tienen mayor rigidez y resistencia.

\section{AGRADECIMIENTOS}

A las fuentes de financiamiento del proyecto de investigación "La tecnología del ferrocemento aplicada en el mejoramiento estructural de muros de adobe, columnas y vigas de concreto en viviendas" claves: CGPI 200326 y SIBEJ 19990504009.

- Instituto Politécnico Nacional, a través del Centro Interdisciplinario de Investigación para el Desarrollo Integral Regional, CIIDIR- Unidad Oaxaca.

- CONACYT a través del Sistema de Investigación Benito Juárez (SIBEJ).

A los ingenieros Tobías Jiménez Ruiz y Margarito Ortiz Guzmán, participantes del proyecto de investigación.

\section{REFERENCIAS}

(1) INEGI. Censo de población y vivienda del estado de Oaxaca INEGI. México, 2000.

(2) Jiménez, T.; Juárez, L.; Caballero, T.: Integración de la vivienda rural y suburbana al medio ambiente. Cuaderno de investigación No. 7. CIIDIR Oaxaca, IPN, México, 1988. 
(3) Morales, V.; Caballero, T.; Jiménez, T.; Ortiz, M. y Juárez, L.: La tecnología del ferrocemento aplicada en el mejoramiento estructural de muros de adobe, columnas y vigas de concreto en viviendas. Informe Técnico final de proyecto de investigación. CGPI 200326. CIIDIR Oaxaca IPN, México, 2002.

(4) ICA-UNAM-SMIE. Edificaciones de mampostería para vivienda. Fundación ICA, A.C. México, 1999

(5) Orduña, A.: "Análisis no lineal de muros de mampostería confinada sujetos a cargas laterales". Engenharia Civil. UM., № 11, 2001.

(6) Gobierno del Distrito Federal. Normas Técnicas Complementarias para Diseño y Construcción de Estructuras de Mampostería. Gaceta Oficial del Distrito Federal. México, 2004.

(7) Organismo Nacional de Normalización y Certificación de la Construcción y Edificación, S.C. NMXC-036-ONNCCE-2004 "Industria de la construcción - Bloques, tabiques o ladrillos, tabicones y adoquines - Resistencia a la compresión - método de prueba". México, 2004.

(8) Organismo Nacional de Normalización y Certificación de la Construcción y Edificación, S.C. NMXC-037-ONNCCE-2005 "Industria de la construcción - Bloques, tabiques o ladrillos, tabicones y adoquines - Determinación de la absorción de agua". México, 2005.

(9) Organismo Nacional de Normalización y Certificación de la Construcción y Edificación, S.C. NMXC-038-ONNCCE-2004 "Industria de la construcción - Determinación de las dimensiones de ladriIlos, tabiques, bloques y tabicones". México, 2004.

(10) Hernández, O.: Tecnología del adobe prensado estabilizado "Tabicote" y normas de diseño. Vivienda Pueblo, S.A. México, 1984.

(11) Merrit, F.: Manual del Ingeniero Civil, Tomo 1, 3ª . Edición, México, 1995.

(12) Juárez, L.: Ventajas del Reforzamiento de muros de adobe como opción para el rescate de viviendas rurales. Tesis de maestría. ITO, Oaxaca, México, 2004.

(13) Meli, R. y Hernández, O.: Propiedades de piezas para mampostería producidas en el D.F. Instituto de Ingeniería UNAM No. 297, México, 1971.

(14) Alcocer, S.: "Comportamiento sísmico de estructuras de mampostería: una revisión. Estado del arte". XI Congreso Nacional de Ingeniería Sísmica. Vol.I Veracruz, México, 1997. 